\title{
Joint Turbo Equalization and Multiuser Detection of MC-CDMA Signals With Low Envelope Fluctuations
}

\author{
Paulo Silva and Rui Dinis, Member, IEEE
}

\begin{abstract}
In this paper, we consider the uplink transmission in multicarrier code-division multiple-access (MC-CDMA) systems. As other multicarrier signals, MC-CDMA signals have high envelope fluctuations and a high peak-to-mean envelope power ratio (PMEPR), which leads to amplification difficulties. This is particularly important for the uplink transmission, since an efficient low-cost power amplification is desirable at the mobile terminals (MTs). Moreover, the transmission over time-dispersive channels destroys the orthogonality between spreading codes, which might lead to significant multiple-access interference levels. To reduce the envelope fluctuations of the transmitted signals, while maintaining the spectral efficiency, the MC-CDMA signal associated to each MT is submitted to a clipping device, followed by a frequencydomain filtering operation. However, the nonlinear distortion effects can be high when an MC-CDMA transmitter with reduced envelope fluctuations is intended (e.g., a small clipping level and/or when successive clipping and filtering operations are employed). In this paper, we define an iterative receiver that jointly performs a turbo multiuser detection and the estimation and cancellation of the nonlinear distortion effects. Our performance results show that the proposed receiver structure allows good performances, very close to the linear receiver ones, even for high system load and/or when a PMEPR as low as $1.7 \mathrm{~dB}$ is intended for each MT.
\end{abstract}

Index Terms-Multicarrier code-division multiple access (MCCDMA), multiuser detection (MUD), nonlinear distortion, turbo equalization.

\section{INTRODUCTION}

$\mathbf{M}$ ULTICARRIER code-division multiple-access (MCCDMA) schemes combine an orthogonal frequencydivision multiplexing (OFDM) modulation [1] with a code-division multiple-access (CDMA) scheme [2]. Spreading is performed in the frequency domain, and MC-CDMA schemes are promising candidates for future broadband wireless systems. Since the transmission over time-dispersive channels destroys the orthogonality between spreading codes, a frequency-domain equalizer (FDE) optimized under an MMSE criterion is usually employed at the receiver [3], [4]. Since an

Manuscript received June 6, 2008; revised October 14, 2008. First published November 25, 2008; current version published May 11, 2009. This work was supported in part by the Fundação para a Ciência e Tecnologia under pluriannual funding, U-BOAT Project PTDC/EEA-TEL/67066/2006, and the FCT/POCI 2010 research Grant SFRH/BD/24520/2005). The review of this paper was coordinated by Dr. C. Cozzo.

P. Silva is with the Escola Superior de Tecnologia, University of Algarve, 8005-139 Faro, Portugal, and also with the Instituto de Telecomunicações, Instituto Superior Técnico, Technical University of Lisbon, 1049-001 Lisbon, Portugal (e-mail: psilva@ualg.pt).

R. Dinis is with the Instituto de Telecomunicações, Instituto Superior Técnico, Technical University of Lisbon, 1049-001 Lisbon, Portugal (e-mail: rdinis@ist.utl.pt).

Color versions of one or more of the figures in this paper are available online at http://ieeexplore.iee.org.

Digital Object Identifier 10.1109/TVT.2008.2010051
MMSE FDE does not perform an ideal channel inversion, we are not able to fully orthogonalize the different spreading codes, which can lead to severe interference levels, particularly for fully loaded systems and/or when different powers are assigned to different spreading codes. To improve the performance, several receivers that perform turbo multiuser detection (MUD) were proposed [5]-[7]. In [5], the use of soft information for interference cancellation is exploited in MC-CDMA systems. An iterative semiblind receiver for coded MC-CDMA systems, able to deal with intracell and intercell interference, is proposed in [6]. A novel low-complexity parallel interference cancellation (PIC) receiver for turbo coded MC-CDMA systems is also investigated in [7]. A promising iterative receiver for multicode MC-CDMA signals was proposed in [8], based on the iterative block decision-feedback equalizer (IB-DFE) concept [9]-[11], allowing significant performance improvements, particularly for fully loaded systems and high spreading factors.

As with other multicarrier schemes, MC-CDMA signals have strong envelope fluctuations and high peak-to-mean envelope power ratio (PMEPR) values, which lead to amplification difficulties. For this reason, it is desirable to reduce the envelope fluctuations of the transmitted signals. This is particularly important for the uplink transmission, since an efficient low-cost power amplification is desirable at the mobile terminal (MT). Several techniques have been recommended for reducing the envelope fluctuations of multicarrier signals [12]-[15]. A promising approach is to employ clipping techniques, combined with a frequency-domain filtering operation so as to reduce the envelope fluctuations of the transmitted signals while maintaining the spectral occupation of conventional schemes [15]. By repeating the clipping and filtering (C\&F) procedure, we can further reduce the PMEPR of the transmitted signals. However, the nonlinear distortion effects can be severe when a transmission with very low PMEPR values is intended [15], [16]. By using iterative receivers with estimation and cancellation of nonlinear distortion effects, we can significantly improve the performance in the presence of strong nonlinear distortion effects [17]-[19]. However, for low SNRs, the error decisions might lead to error propagation effects, since errors in the estimation of nonlinear distortion effects can preclude its cancellation. This is particularly serious for high system load and/or when we decrease the clipping level to further reduce the PMEPR of the transmitted signals [19]. In [16], an enhanced receiver structure for the downlink transmission of MC-CDMA has been considered, where an iterative estimation and cancellation of nonlinear distortion effects is carried out.

In this paper, we consider the uplink transmission in MC-CDMA systems. We modify the approach in [19] so as to 
cope with its major limitations, namely, the poor performance in the presence of severely nonlinear distortion effects and/or at low SNRs, the envelope fluctuation regrowth after the filtering operation, and the limitations of using the soft decisions values to obtain an estimate of the nonlinear distortion effects. To allow an efficient power amplification, the PMEPR-reducing techniques in [15] are adopted by each MT, which can be repeated several times. The base station (BS) has several receive antennas, so as to reduce the transmit power requirements of each MT. To avoid error propagation effects in the typical region of operation, we use channel decoder outputs in the feedback loop, in a turbolike fashion (a similar approach was proposed for OFDM schemes [20]). We define an iterative receiver that jointly performs a turbo MUD and the estimation and cancellation of nonlinear distortion effects, for each iteration, that are inherent to the transmitted signals. To improve the performance at low SNRs, we consider a threshold-based cancellation.

This paper is organized as follows: The linear transmitter and receiver structures are described in Section II. In Section III, we describe the nonlinear transmitter and receiver structures proposed in this paper. Implementation complexity issues are discussed in Section IV. Section V presents a set of performance results, and Section VI is concerned with the conclusions of the paper.

Throughout this paper, we will employ the following notation: bold letters A denote matrices or vectors; $\mathbf{I}_{N}$ denotes the $N \times N$ identity matrix; $\mathbf{0}_{N \times M}$ denotes the $N \times M$ zero matrix; $(\cdot)^{T},(\cdot)^{*},(\cdot)^{H}$, and $\operatorname{diag}(\cdot)$ denote the transpose, conjugate, Hermitian, and diagonal matrices, respectively; $[\mathbf{A}]_{n, m}$ denotes the element of line $n$ and column $m$ of matrix $\mathbf{A}$. $x \bmod y$ is the remainder of division of $x$ by $y$, and $\delta_{k, k^{\prime}}=1$ if $k=k^{\prime}$ and 0 otherwise.

\section{Linear Transmitter ANd Receiver Structures}

In this paper, we consider the uplink transmission in MCCDMA systems employing frequency-domain spreading. The frequency-domain block to be transmitted by the $p$ th MT is $\left\{S_{k, p} ; k=0,1, \ldots, N-1\right\}$, where $N=K M$, with $K$ denoting the spreading factor and $M$ being the number of data symbols for that MT. The frequency-domain symbols are given by $S_{k, p}=\xi_{p} C_{k, p} A_{k \bmod M, p}$, where $\xi_{p}$ is an appropriate weighting coefficient that accounts for the propagation losses, and $\left\{A_{k, p} ; k=0,1, \ldots, M-1\right\}$ is the block of data symbols associated to the $p$ th MT, assumed to be selected from a given constellation (in fact, different constellations could be selected for different data symbols, as when loading techniques are employed; in that case, the power associated to different data symbols is not necessarily the same). $\left\{C_{k, p} ; k=0,1, \ldots, N-\right.$ $1\}$ is the corresponding spreading sequence ${ }^{1}$ (a pseudorandom spreading is assumed, with $C_{k, p}$ belonging to a QPSK constellation; without loss of generality, it is assumed that $\left|C_{k, p}\right|=1$ ).

\footnotetext{
${ }^{1}$ This corresponds to uniformly spreading the chips associated to a given symbol within the transmission band, i.e., to employing a rectangular interleaver with dimensions $K \times M$.
}

As usual, it is assumed that the length of the cyclic prefix (CP) is higher than the length of the overall channel impulse response. We will assume that the $\mathrm{BS}$ has $L$ receive antennas and the received time-domain block associated to the $l$ th diversity branch, after discarding the samples associated to the $\mathrm{CP}$, is $\left\{y_{n}^{(l)} ; n=0,1, \ldots, N-1\right\}$. The corresponding frequencydomain block $\left\{Y_{k}^{(l)} ; k=0,1, \ldots, N-1\right\}$ [i.e., the length$N$ discrete Fourier transform (DFT) of the block $\left\{y_{n}^{(l)} ; n=\right.$ $0,1, \ldots, N-1\}]$ is

$$
\begin{aligned}
Y_{k}^{(l)} & =\sum_{p=1}^{P} S_{k, p} H_{k, p}^{C h(l)}+N_{k}^{(l)} \\
& =\sum_{p=1}^{P} A_{k \bmod M, p} C_{k, p} \xi_{p} H_{k, p}^{C h(l)}+N_{k}^{(l)} \\
& =\sum_{p=1}^{P} A_{k \bmod M, p} H_{k, p}^{(l)}+N_{k}^{(l)}
\end{aligned}
$$

with $H_{k, p}^{C h(l)}$ denoting the channel frequency response between the $p$ th MT and the $l$ th diversity branch at the $k$ th subcarrier, $N_{k}^{(l)}$ being the corresponding channel noise, and $H_{k, p}^{(l)}=$ $\xi_{p} H_{k, p}^{C h(l)} C_{k, p}$. To detect the $k$ th symbol of the $p$ th MT, we will use the set of subcarriers $\Psi_{k}=\{k, k+M, \ldots, k+(K-$ 1) $M$ \}.

By defining $\mathbf{Y}(k)=\left[\begin{array}{lll}\mathbf{Y}^{(1)}(k) & \cdots & \mathbf{Y}^{(L)}(k)\end{array}\right]^{T}$, with $\mathbf{Y}^{(l)}(k)=$ $\left[\begin{array}{lll}Y_{k}^{(l)} & \ldots & Y_{k+(K-1) M}^{(l)}\end{array}\right]$ denoting the line vector with the received samples associated to the set of frequencies $\Psi_{k}$ for the $l$ th antenna, and $\mathbf{A}(k)=\left[\begin{array}{lll}A_{k \bmod M, 1} & \cdots & A_{k \bmod M, P}\end{array}\right]^{T}$, we have

$$
\mathbf{Y}(k)=\mathbf{H}^{T}(k) \mathbf{A}(k)+\mathbf{N}(k)
$$

where $\mathbf{N}(k)=\left[\begin{array}{lll}\mathbf{N}^{(1)}(k) & \cdots & \mathbf{N}^{(L)}(k)\end{array}\right]^{T}$, with $\mathbf{N}^{(l)}(k)=$ $\left[\begin{array}{lll}N_{k}^{(l)} & \cdots & N_{k+(K-1) M}^{(l)}\end{array}\right]$ denoting the line vector with the noise samples associated to the set of frequencies $\Psi_{k}$ for the $l$ th antenna. In (2), $\mathbf{H}(k)$ is the $P \times K L$ overall channel frequency response matrix associated to $\mathbf{A}(k)$, i.e.,

$$
\mathbf{H}(k)=\left[\begin{array}{lll}
\mathbf{H}^{(1)}(k) & \cdots & \mathbf{H}^{(L)}(k)
\end{array}\right]=\left[\begin{array}{lll}
\mathbf{H}_{1}(k) & \cdots & \mathbf{H}_{P}(k)
\end{array}\right]^{T}
$$

where

$$
\mathbf{H}^{(l)}(k)=\left[\begin{array}{ccc}
H_{k, 1}^{(l)} & \cdots & H_{k+(K-1) M, 1}^{(l)} \\
\vdots & & \vdots \\
H_{k, P}^{(l)} & \cdots & H_{k+(K-1) M, P}^{(l)}
\end{array}\right]
$$

is a $P \times K$ matrix with lines associated to the different MTs and columns associated to the set of frequencies $\Psi_{k}$ for the $l$ th antenna, and

$$
\begin{aligned}
& \mathbf{H}_{p}(k)=\left[\begin{array}{lll}
H_{k, p}^{(1)} & \cdots & H_{k+(K-1) M, p}^{(1)}
\end{array}\right. \\
& \left.\begin{array}{llll}
\cdots & H_{k, p}^{(L)} & \cdots & H_{k+(K-1) M, p}^{(L)}
\end{array}\right]^{T}
\end{aligned}
$$

is a column vector associated to the $p$ th MT. 